\title{
Directional PPs and Reference Frames in DRT
}

\author{
Didier Maillat \\ Oxford University and Fribourg University \\ English Linguistics Dept. \\ Miséricorde \\ CH-1700 Fribourg, Switzerland \\ didier.maillatelincoln.ox.ac.uk
}

\begin{abstract}
In this paper, I will argue that the wellknown ambiguity of directional prepositions between the intrinsic and relative readings is not lexical, but can be interpreted as a framework assignment ambiguity of the type observed in the temporal domain. A DRT semantics will then be constructed around a unified model of framework assignment which can be applied across the board to all three universal frame types.
\end{abstract}

\section{Introduction}

A great deal of energy has been spent on the semantic analysis of temporal relations within a sentence and beyond that, between sentences. Crucially, it has been a well-established and intensively studied fact that eventualities (or events) are interpreted within a temporal framework, i.e. a temporal reference frame with respect to which the temporal relations which obtain between those events are interpreted (e.g. Reichenbach 1947, Kamp \& Reyle 1993). For instance, a past tense verb tends, in the absence of any other external clue, to be temporally interpreted with respect to the time of utterance. That is to say that a typical semantic contribution of a simple past is assessed on a twodimensional frame centred on the time of utterance, as is the case in sentence (1), where the past tense is understood as encoding a precedence relation between the event described by the sentence and the time of utterance $n$.

(1) Alice took a walk by the river.

Notice that this is not always the case. In a larger discursive context, a speaker can control some of these parameters, for instance by embedding a simple past within a larger narrative sequence. In this configuration, a simple past is then often interpreted as following the time denoted by the preceding simple past. So, that if we insert (1) within a larger discursive environment, the past tense of took gets interpreted in a different way:

(2) Alice slept in that morning. After a hefty breakfast, she needed some exercise. She [Alice] took a walk by the river.

Although it is still the case that the action described by sentence (1) is located at a time $t$ which precedes $n$, the whole context of (2) adds a further constraint to the interpretation of the past tense in took as it implies a narrative sequence by virtue of which, $t$ has to follow - in the temporal dimension $-t^{\prime}$, the time at which Alice had her breakfast.

In contrast, similar facts in the spatial domain have been barely touched upon by the semanticists' community. In particular, although the existence of spatial reference frames has been ascertained again and again, very little has been done in order to show how, e.g. spatial adverbs or spatial prepositional expressions interact with a spatial framework in order to yield a given meaning. Part of the failure to come up with a satisfactory account can be explained by the relative difficulty to tell the semantic from the pragmatic contributions. It is the goal of this paper to present a formalism that addresses these issues and tries to draw a line between what is encoded and what is contextually derived. In order to do so, I will focus on a set of framework-sensitive PPs, namely directional (or projective) PPs: to the left/right of, in front of I behind, above lbelow and cardinal directions.

Researchers (see for instance Levinson 1996) have shown that human languages make use of only - and up to - three universal reference 
frames in order to express spatial relations. All three of them are instantiated in Indo-European languages. Thus, a single spatial configuration can be expressed in three different ways in these languages, depending on which reference frame is activated. All three examples below are to be interpreted as referring to a unique spatial setting.

(3) Le chat est à l'est de la voiture

(4) the cat is east of the car ABSOLUTE FRAMEWORK

(5) Le chat est derrière la voiture [with respect to the car]

(6) the cat is behind the car INTRINSIC FRAMEWORK

(7) Le chat est à droite de la voiture [with respect to the speaker's viewpoint]

(8) the cat is on the right of the car RELATIVE FRAMEWORK

Crucially, while (5) and (7) - in the relevant readings - are cases of intrinsic and relative encoding, respectively, it should be clear that both sentences fit equally well in the other framework. That is to say that Indo-European languages are systematically ambiguous between the intrinsic and relative reference frames, since directional PPs can be successfully interpreted in both frameworks. Notice that this is also the case in other language families as similar ambiguities are observed in Japanese, Arabic and Sesotho. Most importantly, the spatial area denoted by each of the six directional PPs mentioned above (i.e. all but the cardinal directions) differs depending on the reference frame within which it is interpreted.

The strong claim that the present study will make is that - very much in the fashion of how DRT deals with tenses or temporal adverbials spatial expressions, such as directional prepositions, can be ambiguous between several readings, because these expressions are underdetermined as to how they connect with the spatial framework. And this choice between alternative computations triggers the ambiguity in (5) and (7).

\section{Cross-linguistic Variation}

Also, despite the striking recurrence of these facts throughout the Indo-European languages and other families, it should be pointed out that some languages display other properties. For instance, Tzeltal (Mexico) and Guugu Yimithirr
(Australia) rely (almost) exclusively on the absolute framework. Contrary to what can be observed in Indo-European languages where the absolute frame is relatively rare and used in very restricted contexts. This type of cross-linguistic variation makes up a very strong case in favour of a unified model for all three frame types. If such a model can be constructed, the upshot would consist in being able to account for crosslinguistic variation in terms of parametric setup.

Hausa speakers (Africa), on the other hand, do not apply the Indo-European 'mirror' configuration when they construct a relative frame. In such a language, a tree's front is not facing toward the speaker, but away from her/him (i.e. in a 'tandem' configuration). A suitable model will have to be flexible enough in order to capture this second type of parametric variation in a satisfactory way too.

\section{What's in a framework?}

A first step towards a full understanding of the phenomena described above consists in a thorough analysis of the properties of the three frameworks. In fact, although superficially they appear to be fairly different, the three frameworks are very much alike. In order to see the similarity between them, let us start by assuming a set of conceptual primitives that will make up a spatial framework in the proposed model.

Under the current view, all three frameworks combine three primitives:

(i) a set of three orthogonal axes (frontal, lateral and vertical) in a Euclidean space

(ii) a point of origin

(iii) an orientation vector

Where the orientation vector determines the orientation of the axial system by fixing the direction and orientation of the frontal axis (see below for a detailed explanation of this process).

According to this model any spatial framework is uniquely and sufficiently defined by means of these three elements, only two of which are variables, viz. (ii) and (iii) ${ }^{1}$. In other words, any spatial reference frame is fully de-

\footnotetext{
${ }^{1}$ Strictly speaking, a third parameter is needed in order to fully determine such a space, but, for brevity's sake, I will gloss over the variability of this third parameter and assume that gravity fixes the vertical axis (see Levelt 1996 for further discussion of the issue).
} 
termined if one knows the value of its origin and that of its orientation vector. This means that this is all that is needed in order to evaluate a) the truth-value of a directional expressions in that reference frame and b) to select an appropriate directional expressions for that framework, given some spatial configuration. Notice that contrary to what is suggested in the literature on this topic (e.g. Zwarts \& Winter 2000), the axial structure is not "polarised", in the sense that each axis defines a dimension (frontal, lateral and vertical), but the sides are left unspecified. In other words, the distinction between, e.g. front and back is not built into the system and will have to be handled at a later stage.

To pursue the analogy with the temporal domain, the spatial framework will have to be fixed, by means of assigning a value to the two parameters origin and orientation. Very much like the temporal dimension is organised around its origin, namely the time of utterance $n$ in DRT. Notice, though, that the addition of one more dimension in the spatial domain triggers the need for a second parameter, orientation. And as we will soon find out, a lot, if not all of the computation process needed to interpret a directional preposition goes precisely into the value assignment for the orientation parameter.

However, what we have seen so far is the similarity between the various frameworks, but as we illustrated earlier on, they also differ in a crucial way, which accounts for the ambiguity observed in sentences (5) and (7). A close scrutiny of the three frame types reveals that they differ with respect to their orientation vector. Indeed the computation of the orientation vector appears to constitute the distinguishing factor between the three frames as is shown in table 1 .

\begin{tabular}{|c|c|c|}
\cline { 2 - 3 } \multicolumn{1}{c|}{} & Origin & Orientation \\
\hline Absolute & $\mathrm{G}$ & given \\
Intrinsic & $\mathrm{G}$ & $\mathrm{G}$ \\
Relative & $\mathrm{G}$ & VPT \\
\hline
\end{tabular}

Table 1

Table 1 illustrates that while the orientation parameter of an absolute frame is given as part of the lexical / encyclopaedic knowledge that the speaker has of a preposition like east of, the orientation parameter of an intrinsic frame is defined by the properties of the ground object ( $\mathrm{G}$ in the table). For instance in our example (5), the functional and geometrical properties of the car will yield the corresponding intrinsic orientation. For the relative system, the framework is oriented with respect to a salient viewpoint (VPT), which often coincides with the location of the speaker but may also differ at times. Table 1 also sums up the facts regarding the other parameter, namely the origin. We see that in all cases the origin of the framework is given by the ground object $\mathrm{G}$ (determined by the complement of P). In other words, we can conclude that in the present model, the type of reference frame is fully determined by the sole orientation parameter, which is formally represented by a vector whose origin corresponds to the point of origin of the axial system and whose endpoint is either lexically encoded, or depends on the intrinsic properties of $\mathrm{G}$, or coincides with a salient VPT (viewpoint). In striking contrast with the suggestion made by Crow (1989) who makes use of no less than 4 different variables.

This, however, leaves the question of the actual denotation of directional prepositions open. Building on and extending the kind of vector geometry used above and discussed extensively by O'Keefe (1996) and Zwarts \& Winter (2000), the proposed analysis assumes that a directional preposition denotes an angular range. Crucially, this angular range needs to be fixed or anchored on a framework in order to be fully specified. The model poses that the angular templates are anchored on the reference frame's orientation vector. An obvious advantage in favour of this approach lies in the fact that it readily accounts for the ambiguity observed in (5) and (7), in the sense that a single template can denote two different configurations depending on the value assigned to the orientation vector; hence, the ambiguity. Figure 1 below illustrates the kind of templates that are used in this model (in this case, a bird's eye view of the derrière template). One should point out that the angular range is calculated clockwise from the orientation vector (represented by the arrow in the diagram). 


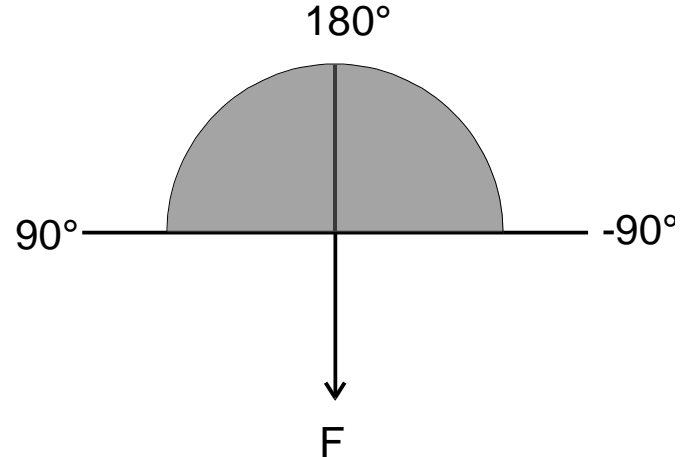

Figure 1

A quick word on the actual values of the angular range displayed here is required. Figure 1 instantiates the maximal denotation of the preposition derrière. That is to say that this template covers the maximal extensional - and truth-functionally correct - scope of the preposition, but it does not reflect the optimal angular range denoted by the preposition (but see Maillat 2000).

One should also point out the obvious relation between the proposed denotation for directional PPs and the denotation suggested by Kamp \& Reyle for temporal adverbials headed by after and before. Thus, they write:

What the phrase after $\alpha$ does is to divide the axis of time into two halves and to say of the described eventuality that it lies in the "upperhalf" ". [1993:626]

Clearly, the same remark applies to the type of directional adverbials under consideration in this paper, as Figure 1 above illustrates. The main difference between the two types of adverbials comes from the dimensionality of the respective domains: while the temporal domain is strictly one-dimensional, space, on the other hand, is three-dimensional. As a result, the purely linear model adopted by Kamp \& Reyle to handle temporal relations is not sufficiently informative to tackle the spatial domain. However, the kind of vector geometry chosen here could of course be successfully applied to the temporal domain. Crucially, decreasing the dimensional arity makes the angular information about vectors irrelevant: in a one-dimensional domain all vectors are parallel to one another. In other words, before and after are the onedimensional equivalents of in front of and behind.

\section{Towards a DRT Model}

In the last part of this paper, I want to look at the kind of formalism that could handle the processes described in the previous sections. In that respect, Discourse Representation Theory seems to be quite appealing as it has been partly developed in order to come to terms with similar framework phenomena in connection with the temporal domain (Kamp \& Reyle 1993 and Van Eijck \& Kamp 1997).

\section{CR.Derrière}

Triggering configuration:

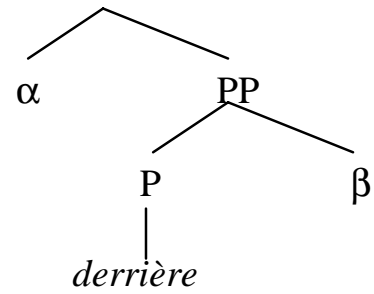

Choose: Orientation point, Opt, from the following items:

(i) $\mathrm{ST}(\beta)$

(ii) ST (salient VPT) \& polarity is switched to negative

where $\mathrm{ST}()$ is an operator which returns the spatio-temporal 'slice' denoted by its argument.

Introduce into $\mathbf{U}_{\mathbf{K}}: 1,1_{1}, 1_{2}$

\section{Introduce into Con $_{\mathrm{K}}$ :}

$$
\begin{aligned}
& 1 \subseteq \mathrm{Opt} \\
& 1_{1}=\mathrm{ST}(\alpha) \\
& 1_{2}=\mathrm{ST}(\beta)
\end{aligned}
$$

$l_{1}$ behind $l_{2} \&$ orientation vector $=\overrightarrow{1_{2}} 1$ \& relational vector $=1_{2} 1_{1} \& 90^{\circ}<\delta<-90^{\circ}$, where $\delta$ obtains between the relational vector and the orientation vector

Perhaps I should first enter a caveat regarding the underlying ontology of this extended model. The extension of the DRT model in order to account for the interpretation of directional prepositional phrases requires that the ontology include some sort of spatial entity. Without further explanation, I introduce locations as a new type of entity. I can only but refer the reader to the relevant literature (Aurnague 
1995 and Asher \& Sablayrolles 1994). An alternative approach would have consisted in leaving out the spatial concepts and manipulating only existing discourse referents with the proviso that some conceptual module à la Jackendoff and/or spatial module would have to translate the DR structures. Here I have opted for a slightly more explicit representation.

The DRT element under scrutiny is the socalled construction rule for the preposition derrière. This choice emphasises the fact that the essential constituent which triggers the setting up of a spatial framework is the spatial adverbial. In this sense, this analysis is very much in line with the treatment of temporal adverbials offered in DRT. To put it slightly differently, it is the directional preposition itself which activates the frame assignment process.

That being said, following the practice of Kamp \& Reyle (1993), I assume that the crucial orientation parameter is encoded as an Orientation Point, Opt, reminiscent of the Temporal Perspective Point and Reference Point (see also the register $r$ in the dynamic version of DRT elaborated by Van Eijck \& Kamp 1997). This choice is pragmatic in nature and, hence, the proposed construction rule is unspecific with respect to the selection process. Consequently, the pragmatic orientation assignment process is appropriately encoded using the Choose operation. Below is a partial quotation, taken from Kamp \& Reyle (1993:621), presenting a typical DRS of a temporal adverbial, which relies on a similar device.

\section{CR.Sunday}

[...]

Choose: Origin of Computation $\mathbf{t}^{\prime \prime}$ from the following items:
(a) $\mathbf{n}$
(b) the current TPpt
(c) The current Rpt [...]

The proposed representation for derrière also makes use of the operator ST(), which extracts the spatio-temporal value of its argument. To be brief, ST() returns a set of ordered pairs of locations and times (Aurnague 1995 and Asher \& Sablayrolles 1994). This step is crucial in order to interpret sentences like

(9) They danced behind the car. involving an event dance (x,e), and in which the adverbial describes the location of the whole activity denoted by the verb, i.e. the dancing. With respect to the original DRT environment, one could think of ST() as being modeled on the dur() operator, which returns the temporal value of its argument.

Most of the semantic work done by the preposition is determined by the set of conditions added to the discourse representation structure. As we can see, the representation proposed here is relatively explicit, and therefore slightly at odds with standard DRT. But as I already argued, this does not constitute a serious obstacle and is meant to illustrate the whole process more clearly. In fact, this series of conditions simply formalises the information contained in the template illustrated in Figure 1, once it has been anchored on a reference frame. It says that the relational vector which obtains between the Ground object and the Figure object must be at an angle $\delta$ from the orientation vector, where $\delta$ is contained in the angular range defined above. If one were to prefer the more implicit kind of representation favoured by Kamp \& Reyle, one should replace the last condition to be introduced in $\mathrm{Con}_{\mathrm{K}}$ with the following one:

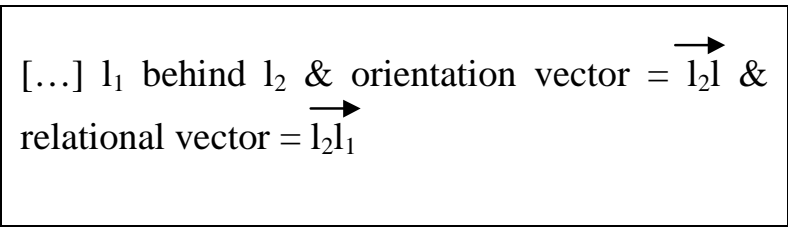

In brief, the semantics of the preposition requires that the figure object be within a determined angular range. Figure 2 below illustrates the kind of calculations needed in order to assess the truth-value of a directional prepositional expression.

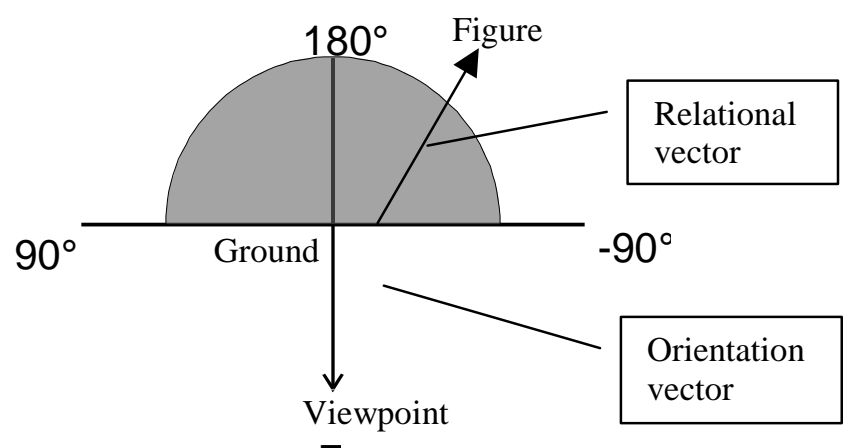

Figure 2 
As we can see, the angle between the Orientation vector and the Relational vector must be within the given range for the prepositional phrase to be true.

Although Figure 2 illustrates a case of relative framework; hence, the 'Viewpoint' parameter. A corresponding figure for an intrinsic framework would be essentially the same, except for the Orientation vector, which would be fixed by the ground object (not a viewpoint).

In this system, the absolute framework does not essentially differ from the other two frame types. The only divergence is that a preposition like east of is not ambiguous between frames. Therefore, its construction rule must reflect the fact that Opt is uniquely determined lexically (at least in Indo-European languages). Thus, CR.east_of does not include a Choose operation, instead a constraint is added to the set of conditions, such that:

$\mathrm{x}$
magnetic north $(\mathrm{x})$
$\mathrm{Opt}:=\mathrm{ST}(\mathrm{x})$

In addition, because the model is applied across the board to all three frameworks, 'absolute' languages like Tzeltal and Guugu Yimithirr do not require any special device in order to be accounted for by the system. Essentially, these languages express directions like we do, they just have stronger selection restrictions on Opt.

Also, Hausa can be readily handled by the theory by means of the polarity index defined in the Choose operation. This pragmatic index keeps track of the orientation assignment process and switches its default (positive) value in case Opt is matched with (ii). Hausa-behind and for that matter, Hausa-in front of-, then, simply makes use of this index to generate the opposite vector from the orientation vector. In Hausa, glossing over the syntactic differences, CR.Hausa_behind would thus be:

\section{[...] Introduce into Con $_{\mathrm{K}}$ :}

$$
\begin{aligned}
& 1 \subseteq \mathrm{Opt} \\
& 1_{1}=\mathrm{ST}(\alpha) \\
& 1_{2}=\mathrm{ST}(\beta)
\end{aligned}
$$

$l_{1}$ behind $l_{2} \&$ orientation vector $=\left\langle\right.$ polarity $>\overrightarrow{1_{2}} 1$ $\&$ relational vector $=1_{2} 1_{1} \& 90^{\circ}<\delta<-90^{\circ}$, where $\delta$ obtains between the relational vector and the orientation vector

The very same formalism elegantly captures the notorious lateral flip observed in IndoEuropean languages. In these languages, the prototypical denotation of to the left of is at a $90^{\circ}$ angle, clockwise, from the front side as defined in a relative framework, but at a $90^{\circ}$ angle, anticlockwise, from the front side as defined in an intrinsic framework. And vice versa for the prototypical denotation of to the right of. That is to say that the lateral axis is, as it were, flipped when you pass from one framework to the other. As a result, in a configuration where the intrinsic and relative denotations of in front of and behind coincide, the denotations of intrinsic to the left of will be that of relative to the right of.

So far, this oddity has been a major obstacle in every formal approach which has tried to tackle the problem. For instance, Zwarts \& Winter (2000) - in the latest attempt to date at formalising the semantics of spatial expressions - have skipped the lateral flip issue and decided to treat only the laterally neutral beside (2000:182). In contrast, the proposed model offers a single treatment to and generalises over the Hausa tandem configuration and the IndoEuropean lateral flip. So that, the very same polarity index will also capture the IndoEuropean lateral flip by generating the opposite vector in case option (ii) is selected in the Choose operation.

Notice that in CR.Derrière, given above, the polarity index is not used in the set of conditions which determine the semantic contribution of the preposition. In other words, derrière is not sensitive to the type of framework in which it is interpreted, i.e. its denotation does not vary across frame types. In the proposed model, this particular behaviour is a direct consequence of the built-in symmetry of the spatial templates. Indeed, as it appears in Figure 1, the defined 
angular range is calculated symmetrically on both sides of the orientation vector.

In fact, there seems to be strong empirical evidence to support this particular property of the model. Thus, the literature on language acquisition and psycholinguistics confirm that the frontal prepositions are acquired faster and are used with fewer mistakes than the lateral pair. In that sense, the spatial templates suggested in this study seem to capture both of these observed facts.

Finally, as is the case in standard DRT with temporal reference points (see Kamp \& Reyle 1993:603), Opt is reset after the triggering clause has been fully processed. This last deletion step is needed so that sentences involving several directional phrases can be treated without imposing the requirement that Opt is held constant throughout, which would clearly be too restrictive. So that, in sequence (10) below, we must be able to assign an intrinsic reading to the first directional PP, while, at the same, we are forced to select a relative reading for the second PP.

(10) I can see three balls. The one in front of the car $[$ Choose $=($ ii) $]$ is identical to the one behind the tree $[$ Choose $=(\mathrm{i})]$.

In other words, the acceptability of (10) under the relevant reading indicates that the orientation assignment process is a "bookkeeping device" which must be reset after the triggering component has been processed (Kamp \& Reyle 1993:603).

\section{Conclusion}

To conclude, I hope to have shown that an elegant DRT model for directional prepositions can be constructed on a single parameter and that this model successfully captures some very puzzling cross-linguistic facts, while, at the same time, shedding some new light on the crucial interaction between spatial expressions and spatial reference frames.

\section{Acknowledgements}

This research project has been supported by a generous grant from the Berrow Trust. I would also like to acknowledge the fruitful feedback received on an earlier version of this paper pre- sented at CSP 2000 in Cambridge; as well as three anonymous reviewers for useful comments.

\section{References}

Asher, N. (1993). Reference to Abstract Objects in Discourse. Kluwer, Dordrecht.

Asher, N. and P. Sablayrolles (1996). A Typology and Discourse Semantics for Motion Verbs and Spatial PPs in French. In: Lexical Semantics (Pustejovsky, J. and B. Boguraev, eds), pp. 163-209, Clarendon Press, Oxford.

Aurnague, M. and L. Vieu (1996). A three-level approach to the semantics of space. In: The semantics of prepositions from mental processing to natural language processing (C. Zelinsky-Wibbelt, ed.), pp. 393-439. Mouton de Gruyter, Berlin.

Aurnague, M. (1995). Orientation in French Spatial Expressions: Formal Representations and Inferences. Journal of Semantics, 12, 239-267.

Bloom, P., Peterson M. A., Nadel L. and Garrett M. F. (eds) (1996). Language and Space. MIT, Cambridge, MA.

Crow, J. (1989). Toward a Semantics for English Spatial Expressions. Ms. PhD Thesis. University of Texas at Austin.

Herskovits, A. (1986). Language and Spatial Cognition: An interdisciplinary study of the prepositions in English. CUP, Cambridge.

Jackendoff, R. (1990). Semantic Structures. MIT, Cambridge, MA.

Levelt, W. J. M. (1996). Perspective Taking and Ellipsis in Spatial Descriptions. In: Language and Space (P. Bloom, M. A. Peterson, L. Nadel and M. F. Garrett, eds), pp. 77-108.

Levinson, S. C. (1996). Frames of reference and Molyneux's question: crosslinguistic evidence. In: Language and Space (P. Bloom, M. A. Peterson, L. Nadel and M. F. Garrett, eds), pp. 109-171.

O'Keefe, J. (1996). The spatial prepositions in English, vector grammar, and the cognitive map theory. In: Language and Space (P. 
Bloom, M. A. Peterson, L. Nadel and M. F. Garrett, eds), pp. 277-316.

Maillat, D. (1999). On the Interpretation of Directional Expressions: Empirical and Theoretical Considerations. Ms. MPhil Thesis. University of Oxford.

Maillat, D. (2000). Which Spatial Template for Directional Prepositions? Ms. University of Oxford.

Nam, S. (1995). The Semantics of locative prepositional phrases in English. Ms. PhD Thesis. University of California, Los Angeles.

Vandeloise, C. (1986). L'espace en français. Seuil, Paris.

Van Eijck, J. and Kamp, H. (1997). Representing Discourse in Context. In: Handbook of Logic and Language (Van Bethem, J. and A. Ter Meulen, eds), pp. 179-237. Elsevier, Amsterdam.

Zwarts, J. and Winter, Y. (2000). Vector Space Semantics: A Model-theoretic Analysis of Locative Prepositions. Journal of Logic, Language and Information, 9/2, 171-213. 